\title{
ON OPTIMAL SIGNALING AND JAMMING STRATEGIES IN WIDEBAND FADING CHANNELS
}

Siddharth Ray

\author{
LIDS, MIT \\ Cambridge, MA 02139, USA \\ Email: sray@mit.edu
}

\author{
Pierre Moulin
}

\author{
Beckman Institute, UIUC \\ Urbana, IL 61801, USA \\ Email:moulin@ifp.uiuc.edu
}

Muriel Médard

\author{
LIDS, MIT \\ Cambridge, MA 02139, USA \\ Email: medard@mit.edu
}

\begin{abstract}
We investigate the fundamental limits on communication performance in wideband fading channels, in the presence of a jammer. We show that energy-limited Gaussian jammers cannot affect the error exponents in the wideband regime. However, if the sender uses an impulsive training scheme, which is close to optimum in the wideband regime, the jammer may be able to interfere with sounding signals if the location of those signals is known. In the latter case, the jammer significantly reduces the wideband capacity limit.
\end{abstract}

\section{INTRODUCTION}

Traditional game-theoretic approaches for coherent additive white Gaussian noise (AWGN) channels with an additive per codeword energy-limited jammer show that a saddle point is reached with a WGN input signal distributions are optimal, while the jammer itself uses additive AWGN to jam the channel. Such approaches readily reveal, in the wideband limit, the fact that an additive jammer with limited per codeword energy cannot affect capacity, since the jammer's contribution to the noise vanishes - the energy per $\mathrm{Hz}$ of the additive noise goes to 0 , while that of the channel's AWGN remains fixed. However, for wideband fading channels, WGN distributions for inputs not only fail to achieve capacity, but, in the limit of infinite bandwidth, provide a null rate $[1,2]$. Instead, the optimum input distributions are peaky: they are characterized by a low duty cycle and highpower symbols $[3,4,5]$.

Questions that naturally arise from the above remarks are the following: in the wideband limit, what effect does a jammer have on capacity and error exponents? Should a jammer still seek to mimic AWGN, as in the coherent case? If not, what strategy should the transmitter adopt to counter the jammer?

We use recent results on capacity-achieving schemes in [8, 10] for low signal-to-noise (SNR) fading channels, which we overview in section 3, to show in [11] that a jammer with no information regarding the transmission scheme has a vanishing effect on capacity in the wideband limit. In this paper, we analyse the error exponents and show, in section 4 , that a Gaussian jammer has no effect on the error exponents. The channel model we use is described in section 2 . In section 5, we show that if the transmitter uses an impulsive training-based scheme, as is often done in practice, the jammer may affect capacity appreciably if it is able to jam the training symbols. We present our conclusions in section 6.

\section{MODEL}

We model the wideband channel as a set of $N$ parallel narrowband channels. In general, the narrowband channels will be correlated. We restrict our analysis in this paper to channels having independent and identical statistics. We also assume that the coherence bandwidth is much larger than the bandwidth of the narrowband channel. Hence, each narrowband channel is modeled as being flat faded. This model is the same as the one used in [8]. Reference [8] shows that low SNR channels are robust to reasonable modeling assumptions. Hence, the results for a more precise channel model may well not differ significantly from that of the simple model we consider in this paper.

Using the sampling theorem, the $m^{\text {th }}$ narrowband channel at symbol time $k$ can be represented as:

$$
\mathbf{y}[k, m]=\mathbf{h}[k, m] \cdot \mathbf{x}[k, m]+\mathbf{w}[k, m]+\mathbf{z}[k, m],
$$

where $\mathbf{h}[k, m], \mathbf{x}[k, m], \mathbf{w}[k, m], \mathbf{y}[k, m]$ and $\mathbf{z}[k, m] \in \mathcal{C}$ are the channel gain, input signal, noise signal, output and jammer signals, respectively, for the $m^{\text {th }}$ narrowband channel at symbol time $k$. These variables take values in the complex plane. The pair $(k, m)$ may be considered as an index for the time-frequency slot, or degree of freedom, to communicate. The fading is Rayleigh, i.e., the channel gain, $\mathbf{h}[k, m]$, is a zero-mean complex Gaussian random variable with independent real and imaginary components. We set the variance of the channel gain to 1 , i.e., $\mathbf{h}[k, m] \backsim$ $\mathcal{C N}(0,1)$. The channel gain is unknown at the transmitter and the receiver. However, its statistics are known to 
both. Hence, we consider a non-coherent channel. The noise signal, $\mathbf{w}[k, m]$, is a zero-mean complex Gaussian random variable with variance 1 . Thus, $\mathbf{w}[k, m] \backsim \mathcal{C N}(0,1)$. Since the narrowband channels are assumed to be independent, we will omit the narrowband channel index, $m$, to simplify notation. The capacity of the wideband channel with average input power constraint, $P$, is thus $N$ times the capacity of each narrowband channel with power constraint $P / N$. We focus on the narrowband channel alone.

We further assume a block fading channel model, i.e., the channel gain is random but fixed for the duration of the coherence time of the channel, and is i.i.d across blocks. Hence, we may omit the time index, $k$, and express the narrowband channel within a coherence block of length $l$ symbols as:

$$
\overline{\mathbf{y}}=\mathbf{h} \overline{\mathbf{x}}+\overline{\mathbf{w}}+\overline{\mathbf{z}}
$$

The input, $\overline{\mathbf{x}}$, satisfies the average power constraint:

$$
\frac{1}{l} E\left[\|\overline{\mathbf{x}}\|^{2}\right]=\text { SNR. }
$$

The jammer also has an average power constraint and spreads its power uniformly across the $N$ narrowband channels. We assume the jammer's signal to be independent across blocks. Hence, the average power constraint on the jammer for each narrowband channel is

$$
\frac{1}{l} E\left[\|\overline{\mathbf{z}}\|^{2}\right]=J \text { SNR. }
$$

where, $J$ is a real and positive constant. As $N$ tends to $\infty$, SNR tends to 0 , and the narrowband channel is in the low SNR regime.

\section{CAPACITY IN THE ABSENCE OF A JAMMER}

In the absence of a jammer, [8,9] show that a non-coherent channel with coherence length

$$
\frac{1}{4} \mathrm{SNR}^{-2 \nu}<l \leq \frac{1}{4} \mathrm{SNR}^{-2(\nu+\epsilon)},
$$

where, $\nu>0$ and $\epsilon \in(0, \min \{1, \nu\})$, has capacity

$C(\mathrm{SNR})=\mathrm{SNR}-\mathrm{SNR}^{1+\min \{1, \nu\}}+O\left(\mathrm{SNR}^{1+\min \{1, \nu\}+\epsilon}\right)$.

The channel capacity and coherence length are related through $\nu$. The expression, $\min \{1, \nu\}$, is the coherence level and is an indicator of the amount of coherence in the channel. A detailed treatment of coherence level can be found in [8].

\subsection{Signaling scheme}

References [8,9] introduce a near-capacity achieving input signaling scheme - the Peaky Gaussian signaling scheme.
We present this scheme here for completeness. For a channel with coherence length

$$
l=\frac{1}{4} \mathrm{SNR}^{-2 \nu}, \quad \nu>0,
$$

transmit in only $\delta(\mathrm{SNR})=\mathrm{SNR}^{1-\min \{1, \nu\}}$ fraction of the blocks. The receiver has perfect knowledge of the blocks used for transmission. Since the power is concentrated in only a fraction of the blocks, the signal to noise ratio for the blocks used for transmission increases to $\mathrm{SNR}_{b}$, where

$$
\mathrm{SNR}_{b} \triangleq \frac{\mathrm{SNR}}{\delta(\mathrm{SNR})}=\mathrm{SNR}^{\min \{1, \nu\}} .
$$

In the blocks chosen for transmission, let $\overline{\mathbf{x}} \sim \mathcal{C N}\left(0, \mathrm{SNR}_{b} I_{l}\right)$. Note that as we increase the coherence length, the fraction of blocks we transmit increases from SNR to 1. Therefore, the signaling changes from a peaky to a continuous one. In this paper, we restrict our attention to the Peaky Gaussian signaling scheme since it achieves the linear as well as the sublinear capacity term, $\mathrm{SNR}^{1+\min \{1, \nu\}}$.

\section{ERROR EXPONENTS}

Now we turn our attention to error probability for peaky signaling schemes. For a coherence block, let

$$
\overline{\mathbf{s}}=\overline{\mathbf{w}}+\overline{\mathbf{z}}
$$

denote the sum of the background noise and jammer's signal. This sum signal is independent across blocks. The distributions over $\overline{\mathbf{x}}$ and $\overline{\mathbf{s}}$ are denoted as $\mathbf{Q}$ and $\mathbf{V}$, respectively. Denote by $R$ the bit rate per narrowband channel per coherence block. The pdfs $\mathbf{Q}$ and $\mathbf{V}$ belong to the feasible sets $\mathcal{Q}(\mathrm{SNR})$ and $\mathcal{V}(J \mathrm{SNR})$, respectively. We use [6] to upper bound the error probability for a coherence block as

$$
P_{e}(\mathbf{Q}, \mathbf{V}) \leq B(\rho, \mathbf{Q}, \mathbf{V}) \triangleq 2^{\rho R} F(\rho, \mathbf{Q}, \mathbf{V}),
$$

for all $\rho \in[0,1]$. In this expression,

$$
F(\rho, \mathbf{Q}, \mathbf{V}) \triangleq \int d \overline{\mathbf{y}}\left[\int d \overline{\mathbf{x}} \mathbf{Q}(\overline{\mathbf{x}}) \mathbf{V}(\overline{\mathbf{y}} \mid \overline{\mathbf{x}})^{\frac{1}{1+\rho}}\right]^{1+\rho}
$$

We further define

$$
\begin{aligned}
E_{r}(\rho, \mathbf{Q}, \mathbf{V}) & \triangleq-\log B(\rho, \mathbf{Q}, \mathbf{V}) \\
& =-\log F(\rho, \mathbf{Q}, \mathbf{V})-\rho R
\end{aligned}
$$

The random coding error exponent when coding is applied across narrowband channels and/or across coherence blocks is given by

$$
E_{r}(R, \mathrm{SNR}, J)=\max _{0 \leq \rho \leq 1} \sup _{\mathbf{Q} \in \mathcal{Q}(\mathrm{SNR})} \inf _{\mathbf{V} \in \mathcal{V}(J \mathrm{SNR})} E_{r}(\rho, \mathbf{Q}, \mathbf{V})
$$


Assuming momentarily that the supremum over $\mathbf{V}$ is achieved by some $\mathbf{V}$, we now ask what special property $\mathbf{V}$ may satisfy? First of all, the optimal $\mathbf{V}$ is generally not memoryless. In the following, we shall be interested in the subset of $\mathcal{V}(J$ SNR $)$ that consists of isotropic pdf's. This set will be denoted by $\stackrel{\circ}{\mathcal{V}}(J$ SNR) and is convex (because the sum of two isotropic functions is an isotropic function). Likewise, we define a feasible set $\stackrel{\circ}{\mathcal{Q}}($ SNR $)$ of isotropic $\mathbf{Q}$. The following lemma shows that if $\mathbf{Q} \in \stackrel{\circ}{\mathcal{Q}}$ (SNR), there is no loss of optimality for the jammer by restricting the maximization of $\mathbf{V}$ over $\stackrel{\circ}{\mathcal{V}}(J$ SNR).

Lemma 1 If $\mathbf{Q}$ is isotropic, then

$$
\sup _{\mathbf{V} \in \mathcal{V}(J \text { SNR })} F(\rho, \mathbf{Q}, \mathbf{V})=\sup _{\substack{\circ \\ \mathbf{V} \in \mathcal{V}(J \text { SNR })}} F(\rho, \mathbf{Q}, \mathbf{V}) .
$$

Proof: Denote by $\mathcal{G}$ the set of $l$-dimensional rotation matrices. For any pdf $\mathbf{V} \in \mathcal{V}(J \mathrm{SNR})$ and $\mathrm{G} \in \mathcal{G}$, define the "rotated pdf"

$$
\mathrm{GV}(\mathbf{v}) \triangleq \mathbf{V}(\mathrm{G} \mathbf{v}) .
$$

Observe that $\mathrm{GV} \in \mathcal{V}(J \mathrm{SNR})$ and that $F(\rho, \mathbf{Q}, \cdot)$ is $\mathcal{G}$ invariant, i.e.,

$$
F(\rho, \mathbf{Q}, \mathbf{V})=F(\rho, \mathbf{Q}, \mathbf{G V}) \quad \forall \mathbf{G} \in \mathcal{G}
$$

Now define the "rotation-averaged" pdf

$$
\underline{\mathbf{V}}(\mathbf{v}) \triangleq \int_{\mathcal{G}} \mathbf{G V}(\mathbf{v}) d \mu(\mathrm{G})
$$

where $\mu$ is the uniform measure on $\mathcal{G}$. Observe that $\underline{\mathbf{V}} \in$ $\stackrel{\circ}{\mathcal{V}}$ (J SNR). We have

$$
\begin{aligned}
F(\rho, \mathbf{Q}, \underline{\mathbf{V}}) & \geq \int_{\mathcal{G}} F(\rho, \mathbf{Q}, \mathbf{G V}) d \mu(\mathbf{G}) \\
& =F(\rho, \mathbf{Q}, \mathbf{V})
\end{aligned}
$$

where the inequality is due to the convexity and the equality to the $\mathcal{G}$-invariance of $F(\rho, \mathbf{Q}, \cdot)$.

Since the negative logarithm of a concave function of $\mathbf{V}$ is a convex function of $\mathbf{V}$, we obtain

Corollary 1 If $\mathbf{Q}$ is isotropic, then

$$
\inf _{\mathbf{V} \in \mathcal{V}(J \mathrm{SNR})} E_{r}(\rho, \mathbf{Q}, \mathbf{V})=\inf _{\mathbf{V} \in \mathcal{V}(J \mathrm{SNR})} E_{r}(\rho, \mathbf{Q}, \mathbf{V})
$$

Let $\tilde{\mathbf{V}}$ denote the radial pdf corresponding to $\mathbf{V}$, i.e.,

$$
\tilde{\mathbf{V}}(\|\mathbf{v}\|)=\left|\mathcal{S}_{l}(\|\mathbf{v}\|)\right| \mathbf{V}(\mathbf{v})
$$

where $\left|S_{l}(r)\right|$ denotes the area of the $l$-dimensional sphere with radius $r$. Likewise, let $\tilde{\mathbf{Q}}$ be the radial pdf corresponding to $\mathrm{Q}$ :

$$
\tilde{\mathbf{Q}}(\|\mathbf{x}\|)=\left|\mathcal{S}_{l}(\|\mathbf{x}\|)\right| \mathbf{Q}(\mathbf{x}) \text {. }
$$

The feasible sets for $\tilde{\mathbf{Q}}$ and $\tilde{\mathbf{V}}$ are convex and are denoted by $\tilde{\mathcal{Q}}(\mathrm{SNR})$ and $\tilde{\mathcal{V}}(J$ SNR $)$, respectively. With a slight abuse of notation, we view the Gallager error exponent as a function of $\tilde{\mathbf{Q}}, \tilde{\mathbf{V}}$ and write

$$
E_{r}(R, \mathrm{SNR}, J)=\max _{0 \leq \rho \leq 1} \sup _{\tilde{\mathbf{Q}} \in \tilde{\mathcal{Q}}(\mathrm{SNR})} \inf _{\tilde{\mathbf{V}} \in \tilde{\mathcal{V}}(J \mathrm{SNR})} E_{r}(\rho, \tilde{\mathbf{Q}}, \tilde{\mathbf{V}}),
$$

where, $E_{r}(\rho, \tilde{\mathbf{Q}}, \tilde{\mathbf{V}})$ is concave in $\tilde{\mathbf{Q}}$ and convex in $\tilde{\mathbf{V}}$. This leads to the following results:

- For each value of $\rho$, the payoff function $E_{r}(\rho, \tilde{\mathbf{Q}}, \tilde{\mathbf{V}})$ admits a saddlepoint.

- If the jammer selects a Gaussian pdf, the Gaussian background noise dominates the jammer's noise (JSNR « 1 ), and so the jammer's effect on error exponent vanishes with increasing bandwidth.

We conjecture that

$$
\begin{aligned}
& \sup _{\mathbf{Q} \in \mathcal{Q}(\mathrm{SNR})} \inf _{\mathbf{V} \in \mathcal{V}(J \mathrm{SNR})} E_{r}(\rho, \mathbf{Q}, \mathbf{V}) \\
& =\sup _{\mathbf{Q} \in \stackrel{\mathcal{Q}}{\text { (SNR })} \mathbf{V} \in \mathcal{V}(J \mathrm{SNR})} \inf _{r} E_{r}(\rho, \mathbf{Q}, \mathbf{V}),
\end{aligned}
$$

and hence, there is no loss of optimality for the transmitter to use an isotropic strategy. This follows from the saddlepoint property above.

As a first step in exploring numerical algorithms for solving the minimization problem (1), we consider the singleparameter family (defined below with parameter $\epsilon \in(0,1])$ : Assume the sender selects a Gaussian strategy and the jammer selects a pulsed Gaussian strategy, i.e., for some $\epsilon \in$ $(0,1]$,

$$
p_{\mathbf{Z}}(\mathbf{z})=(1-\epsilon) \delta(\mathbf{z})+\epsilon p_{\mathbf{Z}^{\prime}}(\mathbf{z}),
$$

where, $\delta(\mathbf{z})$ is the $l$-dimensional Dirac impulse and $\mathbf{Z}^{\prime}$ is i.i.d. Gaussian with zero mean and variance $\frac{1}{\epsilon} J$ SNR. Let the optimal value of $\epsilon$ be $\epsilon^{*}$ :

Now, as SNR $\rightarrow 0, \epsilon^{*} \rightarrow 1$, (proof omitted here for brevity) i.e., a pulsed Gaussian strategy is not useful to a hypothetical powerful jammer that could dominate background noise. Also, for any $J, \epsilon^{*}$ is bounded away from 0 . This suggests a pulsed Gaussian strategy may not be terribly useful for the jammer.

\section{EFFECT OF A JAMMER ON A TRAINING BASED SCHEME}

Zheng et al. [8] show that in the absence of a jammer, the linear capacity term, SNR, is achievable when training is performed in the blocks chosen for transmission. This training scheme is described later on in this section. Hence, in the absence of a jammer, the wideband capacity limit is achievable with training. 
We now consider the effect of a Gaussian jammer on capacity when the training scheme is used. The jammer is assumed to know which blocks are used for transmission and spreads its energy equally over them. He does not spend any energy on the blocks not used for transmission. He also knows the location of the training symbol within each block and uses all its available energy for this block,

$$
\mathrm{E}_{\text {jammer }}=l J \mathrm{SNR}_{b},
$$

to jam the training symbol. In this section, we confine our analysis to $J=1$. The data symbols are therefore not affected by the jammer. The transmitter is aware of the existence of the jammer and, to compensate, it adjusts its energy allocation between training and data transmission.

We now describe the training scheme. In a coherence block used for transmission, the first symbol is used for training and the rest for data transmission. We rewrite the channel model as

$$
\begin{aligned}
& \mathbf{y}_{1}=\mathbf{h} \mathbf{x}_{1}+\mathbf{w}_{1}+\mathbf{z}_{1}, \\
& \mathbf{y}_{i}=\mathbf{h} \mathbf{x}_{i}+\mathbf{w}_{i}, \quad i=2, \ldots, l .
\end{aligned}
$$

and, $\mathbf{z}_{1} \backsim \mathcal{C N}\left(0, E_{\text {jammer }}\right)$. The total energy available in the block for the transmitter is

$$
\mathrm{E}_{\mathrm{total}}=l \mathrm{SNR}_{b} .
$$

The first symbol of the block is used for training using $\gamma \in$ $(0,1)$ fraction of the total energy. The remaining fraction is used for communicating data. The energy used for training is $\gamma \mathrm{E}_{\text {total }}=\gamma l \mathrm{SNR}_{b}$, and the training symbol is $\mathbf{x}_{1}=$ $\sqrt{\gamma \mathrm{E}_{\text {total }}}$. The receiver computes the minimum mean-squares error (MMSE) estimate of $\mathbf{h}$ from $\mathbf{y}_{1}$. Using $\hat{\mathbf{h}}$ and $\tilde{\mathbf{h}}$ to denote the estimate and estimation error of $\mathbf{h}$, respectively, we have:

$$
\begin{aligned}
\mathbf{h} & =\hat{\mathbf{h}}+\tilde{\mathbf{h}} \\
\hat{\mathbf{h}} & \sim \mathcal{C N}\left(0, \frac{\frac{\gamma E_{\text {total }}}{1+E_{\text {jammer }}}}{1+\frac{\gamma E_{\text {total }}}{1+E_{\text {jammer }}}}\right), \\
\tilde{\mathbf{h}} & \sim \mathcal{C N}\left(0, \frac{1}{1+\frac{\gamma \mathrm{E}_{\text {total }}}{1+E_{\text {jammer }}}}\right) .
\end{aligned}
$$

Since, $\mathbf{h}$ is Gaussian, $\hat{\mathbf{h}}$ and $\tilde{\mathbf{h}}$ are independent Gaussian. Note that the jammer's effect is to increase the estimation error.

For the remaining $l-1$ symbols within the same block, (1$\gamma) \mathrm{E}_{\text {total }}=(1-\gamma) l \mathrm{SNR}_{b}$ energy is used to send data using an i.i.d Gaussian code. The channel in this phase can be represented as

$$
\mathbf{y}_{i}=\hat{\mathbf{h}} \mathbf{x}_{i}+\underbrace{\tilde{\mathbf{h}} \mathbf{x}_{i}+\mathbf{w}_{i}}_{\mathbf{v}_{i}}, \quad i=2, \ldots, l
$$

$\mathbf{x}_{i}$ are i.i.d complex Gaussian random variables, $\mathbf{x}_{i} \sim \mathcal{C N}\left(0, \sigma_{x}^{2}\right)$, where

$$
\sigma_{x}^{2}=\frac{(1-\gamma) l \mathrm{SNR}_{b}}{(l-1)}
$$

$\tilde{\mathbf{h}} \mathbf{x}_{i}$ is the noise due to the estimation error from the training phase coupled with the input signal. Combining the additive white noise with the noise due to estimation error, we have

$$
\mathbf{v}_{i} \triangleq \tilde{\mathbf{h}} \mathbf{x}_{i}+\mathbf{w}_{i}
$$

Note that $\mathbf{v}_{i}$ is uncorrelated with, but not independent of $\hat{\mathbf{h}} \mathbf{x}_{i}$. It is zero-mean and has a variance of

$$
E\left[\left|\mathbf{v}_{i}\right|^{2}\right]=E\left[|\tilde{\mathbf{h}}|^{2}\right] E\left[\left|\mathbf{x}_{i}\right|^{2}\right]+E\left[\left|\mathbf{w}_{i}\right|^{2}\right] \triangleq \sigma_{v}^{2} .
$$

Let us define $f^{*}(\mathrm{SNR})$ as

$$
f^{*}(\mathrm{SNR}) \triangleq \max _{\gamma \in(0,1)}\left[\frac{E\left[|\hat{\mathbf{h}}|^{2}\right] \sigma_{x}^{2}}{\sigma_{v}^{2}}\right] .
$$

If we assume $\mathbf{v}_{i}$ to be Gaussian and independent of the input signal, we can lower bound the capacity of the training based scheme, $C_{T}$ (SNR), using [7],

$$
\begin{aligned}
& C_{T}(\mathrm{SNR}) \\
& \quad \geq \delta(\mathrm{SNR}) \cdot \frac{l-1}{l} \cdot \max _{\gamma \in(0,1)} E_{\hat{\mathbf{h}}}\left[\log \left(1+\frac{|\hat{\mathbf{h}}|^{2} \sigma_{x}^{2}}{\sigma_{v}^{2}}\right)\right] \\
& \quad=\delta(\mathrm{SNR}) \cdot\left[f^{*}(\mathrm{SNR})+o\left(f^{*}(\mathrm{SNR})\right)\right] .
\end{aligned}
$$

The following lemma is the computation of $f^{*}(\mathrm{SNR})$. The proof is omitted from this paper for brevity.

\section{Lemma 2}

$$
\begin{aligned}
& f^{*}(\mathrm{SNR})=(\sqrt{2}-1)^{2} \cdot \mathrm{SNR}^{\min \{1, \nu\}}+o\left(\mathrm{SNR}^{\min \{1, \nu\}}\right), \\
& \arg \max _{\gamma \in(0,1)} E_{\hat{\mathbf{h}}}\left[\log \left(1+\frac{|\hat{\mathbf{h}}|^{2} \sigma_{x}^{2}}{\sigma_{v}^{2}}\right)\right]=(\sqrt{2}-1)+o(1) .
\end{aligned}
$$

Combining this lemma with (2), we obtain the following lower bound to the capacity using the training based scheme:

$$
C_{T}(\mathrm{SNR}) \geq(\sqrt{2}-1)^{2} \cdot \mathrm{SNR}+o(\mathrm{SNR}) .
$$

\subsection{Discussion}

We see from (3) that when a training based scheme is used and the jammer jams only the training symbol, the linear capacity term is reduced from SNR to $(\sqrt{2}-1)^{2}$ SNR. Hence, the jammer reduces the wideband capacity limit by approximately $83 \%$ when the training scheme is used. 
The jammer increases the variance of the channel estimation error which results in an increase in the "noise" component of the received signal, during the data transmission phase. This leads to a reduction of the wideband capacity limit. In the absence of the jammer, [8] shows that the fraction of energy required for training goes to 0 as SNR tends to 0 . In the presence of the jammer, the optimal energy required for training does not go to 0 and is $\sqrt{2}-1 \sim 41 \%$ of the total block energy.

\section{CONCLUSIONS}

We have shown that a jammer using a Gaussian transmission strategy does not, in principle, have the ability to reduce error exponents for fading channels in the wideband regime. The presence of fading does affect, to some extent, the ability of the transmitter to counter the jammer in the wideband limit. An attractive means of approaching the wideband capacity in the absence of jamming, impulsive training schemes, is affected by jamming if the location of the training signals is known by the jammer. A safer strategy, therefore, is to use a peaky signaling strategy that does not rely on the use of training sequences or, to use randomized training [11], which makes it difficult for the jammer to effect channel state estimation.

\section{Acknowledgements}

The authors acknowledge the NSF grant: ANI-0335256.

\section{References}

[1] M. Médard and R. G. Gallager, " Bandwidth Scaling for Fading Multipath Channels", IEEE Transactions on Information Theory, 48(4):840-852, April 2002.

[2] V. G. Subramanian and B. Hajek, "Broad-band fading channels: signal burstiness and capacity", IEEE Trnsactions on Information Theory, Volume: 48 Issue: 4 , April 2002 Page(s): 809 - 827.

[3] R. S. Kennedy, Fading Dispersive Communication Channels, Wiley Interscience, New York, NY, 1969.

[4] I. E. Telatar and D. N. C. Tse, "Capacity and mutual information of wideband multipath fading channels", IEEE Transaqctions on Information Theory, 46(4):1384-1400, July 2000.

[5] S. Verdú, "Spectral efficiency in the wideband regime", IEEE Transactions on Information Theory, 48(6):1319 -1343, June 2002.
[6] R. G. Gallager, "A Simple Derivation of the Coding Theorem and Some Applications", IEEE Transactions on Information Theory, Vol. 11, No. 1, pp. 3-18, January 1965.

[7] M. Médard, "The effect upon channel capacity in wireless communications of perfect and imperfect knowledge of the channel", IEEE Transactions on Information Theory, Vol. 46, May 2000.

[8] L. Zheng, D. N. C. Tse and M. Médard, "Channel Coherence in the low SNR Regime", submitted to IEEE Transactions on Information Theory. Available at http://web.mit.edu/lizhong/www/.

[9] S. Ray, M. Médard and L. Zheng, "Wideband Noncoherent MIMO Capacity, ISIT 2005, Adelaide, Australia, Sep. 4-9, 2005.

[10] S. Ray, M. Médard and L. Zheng, "On Non-coherent MIMO Channels in the Wideband Regime: Capacity and Reliability", submitted to IEEE Transactions on Information Theory. Available at ArXiv cs.IT/0603018.

[11] S. Ray, P. Moulin and M. Médard, "On Jamming in the Wideband Regime ", ISIT 2006, Seattle, WA, July 2006. 